\title{
ARTICLE
}

\section{Altered spinal cord activity during sexual stimulation in women with SCI: a pilot fMRI study}

\author{
Marcalee Alexander ${ }^{1,2,3}$, Natalie Kozyrev ${ }^{4,5}$, Chase R Figley ${ }^{6,7,8}$ and J Scott Richards ${ }^{1}$
}

INTRODUCTION: The objective of this study was to assess the feasibility of the use of functional magnetic resonance imaging (fMRI) to evaluate the spinal activation during sexual response of the thoracic, lumbar and sacral spinal cord.

MATERIALS AND METHODS: This is a laboratory-based pilot study in human females at a University-based medical center in the United States. In three healthy spinal cord injury $(\mathrm{SCl})$ females, spinal cord activations during sexual audiovisual stimulation (alone), genital self-stimulation (alone) and simultaneous audiovisual and genital self-stimulation (combined) were assessed and then compared with each subjects' remaining sensory and motor function.

RESULTS: Spinal fMRI responses of the intermediolateral columns were found during audiovisual stimulation in both subjects with incomplete injuries, but they were not observed in the subject with a complete injury. Moreover, sacral responses to combined stimulation differed greatly between the subjects with complete and incomplete injuries.

CONCLUSION: These results not only provide the first in vivo documentation of spinal fMRI responses associated with sexual arousal in women with SCls, but also suggest that spinal cord fMRI is capable of distinguishing between injury subtypes. Therefore, although there are certain limitations associated with $\mathrm{fMRI}$ during sexual stimulation (for example, movement artifacts, an artificially controlled environment and so), these findings demonstrate the potential utility of incorporating spinal cord fMRI in future research to evaluate the impact of specific patterns of $\mathrm{SCl}$ on sexual responses and/or the effects of treatment.

Spinal Cord Series and Cases (2017) 3, 16041; doi:10.1038/scsandc.2016.41; published online 2 February 2017

\section{INTRODUCTION}

Sexuality is a high priority for women with tetraplegia and paraplegia. ${ }^{1}$ However, despite women's interest, they still report diminished sexual satisfaction and activity after injury. ${ }^{2}$ Specific impacts of spinal cord injury $(\mathrm{SCl})$ on sexual responses ${ }^{3-5}$ have been documented, and methods have been developed to analyze these responses. ${ }^{6,7}$ Nevertheless, many women with $\mathrm{SCl}$ remain troubled by sexual dysfunctions.

In order to best understand the mechanisms associated with sexual dysfunctions that occur after $\mathrm{SCl}$ and thus develop new treatments, it is important to understand how injury affects sexual responses. Laboratory studies in women with specific levels and degrees of SCls have been used to map the neurological control of sexual responses, and the peripheral and genital responses of women with $\mathrm{SCls}$ have been assessed in response to sexual arousal and orgasm. However, although animal studies have documented the location of spinal neurons activated in response to pelvic and pudendal nerve stimulation ${ }^{8}$ and the impact of somatosensory stimulation on both vaginal blood flow and the urethrogenital reflex, ${ }^{9}$ we have yet to observe the actual neurological events occurring in the spinal cords of women with $\mathrm{SCl}$. Therefore, building on recent studies using functional magnetic resonance imaging (fMRI) to study spinal cord responses to sexual stimulation in able-bodied men ${ }^{10}$ and women, ${ }^{11}$ this case series is aimed at determining the feasibility of performing spinal cord fMRI on women with SCls and whether this approach can be utilized in future studies to assess sexual responses among SCl patients.

\section{MATERIALS AND METHODS}

Subjects

Subjects included three healthy premenopausal females with SCls. Two participants (Subjects 1 and 2) had American Spinal Injury Association (ASIA) Impairment Scale (AIS) D SCls at the C5 and C5R and C6L levels, respectively, whereas the third participant (Subject 3) had a T10 AIS A injury. All subjects underwent a complete International Neurologic Standards for SCl Assessment performed by a trained physician in addition to detailed medical and sexual history related to preinjury and postinjury functioning. This study was approved by the Institutional Review Board of the University of Alabama at Birmingham School of Medicine, and all subjects provided written informed consent prior to enrollment.

\begin{abstract}
Sexual stimulation protocols
Subjects participated in three consecutive sexual stimulation protocols while laying supine inside the bore of a whole-body 3 T MRI scanner, and complete details of these protocols have been previously reported. ${ }^{11}$ Briefly, the goal of the first protocol was to observe subjects' spinal cord response to sexual psychogenic audiovisual stimulation (i.e., erotic films). The second protocol was performed to assess each subject's spinal cord response to manual self-stimulation (that is, clitoral stimulation); and the
\end{abstract}

${ }^{1}$ Department of Physical Medicine and Rehabilitation, University of Alabama at Birmingham, Birmingham, AL, USA; ${ }^{2}$ Rehabilitative Medicine, Birmingham VA Medical Center, Birmingham, AL, USA; ${ }^{3}$ Department of Physical Medicine and Rehabilitation, Harvard School of Medicine, Boston, MA, USA; ${ }^{4}$ Centre for Neuroscience Studies, Queen's University, Kingston, ON, Canada; ${ }^{5}$ Department of Molecular Medicine and Robarts Research Institute, Western University, London, ON, Canada; ${ }^{6}$ Department of Radiology, University of

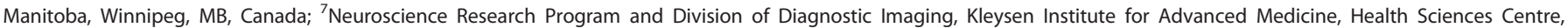
Winnipeg, MB, Canada and ${ }^{8}$ Department of Psychological and Brain Sciences, Johns Hopkins University, Baltimore, MD, USA.

Correspondence: M Alexander (spinalcordmd@live.com)

This study was previously presented in a different format at ISCOS in Maastricht.

Received 2 September 2016; accepted 11 December 2016 
third protocol aimed to elicit maximal spinal sexual responses to combined audiovisual and manual sexual stimulation (that is, erotic films and clitoral stimulation).

\section{Imaging protocol}

Details of the imaging protocol have been previously described, and the reader is referred to the original article ${ }^{10}$ for this information. Briefly, however, imaging was performed on a $3 \mathrm{~T}$ whole-body MRI system (Achieva, Philips Healthcare, Best, The Netherlands). During each experiment, subjects were carefully aligned and centered at T12 on a phased-array spine coil, and peripheral pulse measurements were monitored using an optical sensor attached to the second digit of the resting, non-dominant hand.

To reduce the likelihood and severity of motion artifacts, MRI images were analyzed as previously reported. ${ }^{10}$ In addition, subjects were instructed to remain still during the study. Masking tape was applied across the subject's torso and abdomen to increase their awareness of motion, and the occurrence of orgasm was taken into account during data analysis.

\section{RESULTS}

Subject 1 was a 43-year-old married Caucasian female, 26 years after motor vehicle accident with resultant C5 AIS D tetraplegia. Medical history was remarkable for tubal ligation, endometriosis and breast implants. Significant medications included ditropan and baclofen. She reported full bladder control along with normal bowel control. Sexual function was remarkable for complaints of increased time to orgasm, no complaints of arousal dysfunction, decreased sexual desire over the past few years and no pain with intercourse. Muscle testing revealed grade 5 strength throughout the left lower extremity with grades $2-3$ strength throughout the right lower extremity. Combined pinprick and light touch score was 28 at T11-L2 (maximum possible is 32 ), whereas at S3-5 there was a combined sensory score of 12 . Pinprick was preserved on the right side but not on the left. Bulbocavernosus and anal wink reflexes were intact, and the subject had voluntary rectal contraction.

During audiovisual sexual stimulation, imaging of the thoracolumbar spinal cord revealed increased activity in the left intermediolateral (IML) column, right and left dorsal horn and dorsal commissural nucleus (DCN). In the lumbosacral area, there was increased activity in the left ventral horn, left dorsal horn and possibly in the sacral parasympathetic nucleus, in addition to decreased activity in the right ventral horn. During self-stimulation, thoracolumbar imaging revealed increased activity in the DCN, right ventral horn and possible decreased activity in the left dorsal horn. In the lumbosacral area, there was decreased activity in the left ventral horn and possibly in the dorsal gray commissure (however, it should be noted that these changes could also be related to movement artifacts). During combined stimulation, there was increased activity in the left IML cell column and decreased activity in the left dorsal horn and right ventral horn in the thoracolumbar area. In the lumbosacral area, there was decreased activity in the right ventral horn, sacral parasympathetic nucleus and left dorsal horn.

Subject 2 was a 50-year-old married Caucasian female with a 33-year history of C5/6 incomplete tetraplegia. Medical history was not significant nor were medications. She reported partial control of bladder and bowel function. She denied any issues with sexual arousal, desire, orgasm or pain. Muscle testing revealed grade 4 strength throughout both lower extremities with intact voluntary rectal contraction. She had combined pinprick and light touch sensory score of 25 in the T11-L2 dermatomes with preservation of greater sensation on the left, and a total sensory score of 13 at S3-5 with greater preservation on the left. She had intact bulbocavernosus and anal wink reflexes.

During the audiovisual stimulation session, in the thoracolumbar area there was increased activity in the DCN, throughout the left IML column, dorsal horn and ventral horn and decreased activity in the DCN. In the lumbosacral area, there was increased activity in the dorsal gray commissure, left sacral parasympathetic nucleus with decreased activity in the right sacral parasympathetic nucleus and right dorsal horn. This was consistent with her pattern of injury, where the neural circuits mediating psychological responses were partially spared. During the manual stimulation session, there was increased activity in the right IML column along with decreased activity in the ventral and dorsal horns at the thoracolumbar levels; however, movement artifacts were too severe to accurately characterize activations throughout the lumbosacral regions of the cord. Similarly, the patient reached orgasm during the combined audiovisual and self-stimulation protocol, and movement artifacts were too severe to accurately assess which areas were active during orgasm.

Subject 3 was a 34-year-old single African-American female who sustained a gunshot wound with resultant T10 AIS A SCI 17 years prior. Her sensation was intact to T10 and absent below. She had a bulbocavernosus and anal wink reflex but no voluntary rectal contraction. The subject had no significant medical history and was not on any medications. She reported no control of bladder function and partial control of bowel function. She also denied pain, desire or arousal issues and had orgasms but complained that they took too long to occur.

In the audiovisual stimulation protocol, Subject 3 demonstrated minimal decrease in activity in the left IML column and DCN along with minimal activity in the right dorsal horn in the sacral area. This lack of overall activity is consistent with the patient's functional deficit to psychogenic arousal. In the self-stimulation protocol, there was increased activity in the thoracic area in the left dorsal horn and IML column area along with increased activity in the left sacral parasympathetic nucleus. During the audiovisual plus self-stimulation protocol, there was increased activity in the DCN of the thoracolumbar area along with increased activity in the dorsal gray commissure in the sacral area.

In comparing these three subjects' responses on fMRI (Figure 1), the subject with a complete injury had less change in neurological activity in the thoracolumbar area in response to audiovisual stimulation than the other subjects had, suggesting that both subjects with incomplete injuries had spared descending psychogenic inputs. Similarly, responses to self-stimulation differed between the subject with complete T10 injury and the incomplete subjects in the thoracolumbar area; however, the sacral area responses appeared to be similar between complete and incomplete subjects, as would be expected because all subjects had intact sacral reflex functions. During the final combined stimulation session, two subjects achieved orgasms, one with a complete injury and one with an incomplete injury. These findings support the concept that under normal conditions the sacral parasympathetic nucleus receives tonic descending inhibition, presumably from the brainstem, and that when this pathway is disrupted by injury, orgasmic function will remain as long as sacral reflexes are present.

The responses of subjects with $\mathrm{SCls}$ were observationally different than responses of able-bodied women. ${ }^{11}$ During psychogenic audiovisual stimulation, both subjects with incomplete injuries demonstrated positive responses in the IML cell column, similar to healthy controls. However, DCN activations were notably absent for Subjects 1 and 3, sacral activity was different than controls for Subjects 1 and 2 and completely absent for Subject 3. During the reflexogenic genital self-stimulation condition, all three patients' thoracolumbar responses differed from that of healthy controls, with positive and negative fMRI responses observed throughout the thoracolumbar cord segments; however, consistent with controls, all three subjects demonstrated negative signal intensity changes throughout the sacral cord segments. Finally, during the combined psychogenic/ reflexogenic stimulation, all three subjects demonstrated similar 
Human Thoracolumbar Spinal Cord (T/L)

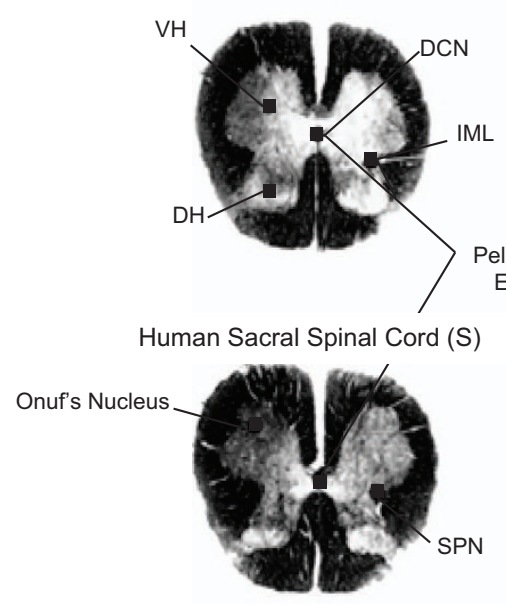

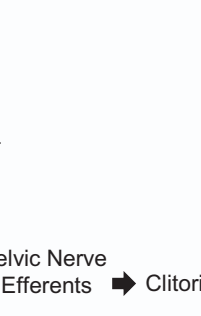

Healthy Controls SCI Subject 1 $\mathrm{N}=9$

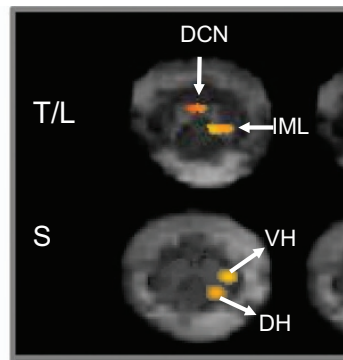
C5, AIS D SCI Subject 3 Condition 1: AudioVisual (AV)
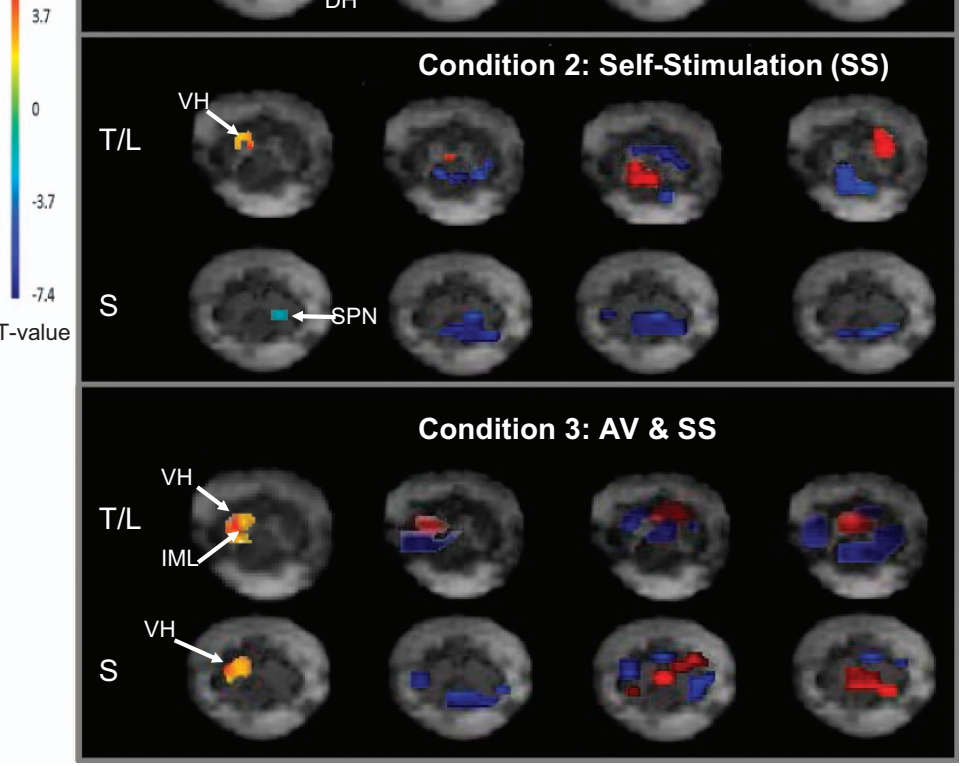

Figure 1. Spinal cord fMRI responses to varying types of sexual stimulation.

responses throughout the thoracolumbar cord, which were not dissimilar from healthy controls, whereas the sacral cord responses for all three subjects differed greatly compared with healthy controls.

Different spinal cord responses were observed within each individual subject in response to audiovisual versus manual versus combined stimulation conditions. However, although we anticipated some interactions between successive conditions, responses to combined physical and psychological stimuli show a clearly distinct pattern of active regions from that seen in pure audiovisual and pure manual stimulation, consistent with the complex interplay of these two components of the sexual response.

\section{DISCUSSION}

Although sexual dysfunction is a highly prevalent and devastating consequence of $\mathrm{SCl}$, relatively little is known about how human spinal cord pathways and neural function are disrupted by injury. Our recent spinal cord fMRI study of healthy women demonstrated that psychological (psychogenic) and physical (reflexogenic) aspects of sexual arousal are anatomically distinct, with the psychogenic activations occurring primarily in the IML cell column and the DCN of the thoracolumbar cord and reflexogenic activations occurring throughout right lumbar cord segments. ${ }^{11}$ However, although relationships between the level/severity of $\mathrm{SCl}$ and the preservation of sensation and sexual functioning have been previously reported in women, ${ }^{5}$ and animal injury models have suggested the involvement of various spinal cord nuclei, $^{12}$ the case studies reported herein and their associated imaging findings represent (to the best of our knowledge) the first glimpses of neural processing in the spinal cords of women with SCls in response to different types of sexual arousal.

By measuring $\mathrm{fMRI}$ responses throughout thoracic, lumbar and sacral spinal cord segments during psychogenic, reflexogenic and combined components of the sexual response, we report, for the first time, the neural correlates of autonomic and sexual responses in three women with different types of $\mathrm{SCl}$ (that is, two women with incomplete AIS D injuries and one with a complete T10 injury), and how these differed compared with each other and to neurologically healthy control subjects. In the case of psychogenic stimulation, there was very little activity in the thoracolumbosacral cord for the woman with the complete injury compared with those subjects with incomplete injuries. In contrast, findings during manual stimulation were similar between all subjects. This would be expected because reflex sacral pathways were intact in all subjects.

The manual and combined stimulation results revealed significant movement artifact in all subjects, and thus these data are difficult to interpret. However, the two subjects who experienced orgasm appeared to demonstrate increased overall activity in the spinal cord compared with the subject who did not experience orgasm. This would also be expected owing to the reflex nature of orgasm causing motion artifacts that may have masked signal changes in the spinal cord related specifically to orgasm.

Although these findings are preliminary and should therefore not be over interpreted or broadly generalized, they demonstrate the feasibility of correlating fMRI data with physical and historical findings from persons with $\mathrm{SCls}$ as well as the difficulty of documenting those data during movement. The understanding of sexual responses in women and men with $\mathrm{SCls}$ has greatly increased over the past 20 years, ${ }^{13,14}$ and this work represents 
another step in the documentation of the neurophysiology of sexual responses. We know from previous laboratory studies that women and men with the preservation of the combined ability to perceive pinprick and light touch sensation in the T11-L2 dermatomes are more likely to demonstrate psychogenic arousal $^{5,15}$ and that orgasmic function through genital stimulation is possible in women and men with preservation of genital sensation and/or preservation of genital reflexes. ${ }^{5,14,16}$ Yet, we do not have definitive proof of the neurological pathways involved in those responses. Based upon the peripheral physical events associated with sexual response, it follows that orgasmic sensation is chronologically related to a generalized sympathetic response followed by a parasympathetic response. At the level of brain, Georgiadis et al. ${ }^{17}$ demonstrated that there was similar activation between men and women in the cerebellar vermis, anterior lobe on the left along with conjoint activation in the region of the deep cerebellar nuclei extending anteriorly to include the pontine tegmentum. In women, there was also increased activity in the right insula as compared with men. Interestingly, in both sexes there was deactivation in the left ventromedial and orbitofrontal cortex during orgasm. Neuroplasticity in sympathetic pathways ${ }^{18}$ has been shown after $\mathrm{SCl}$ and it is possible that sympathetic pathways facilitate neurological transmission of impulses to the brain, whereas reflex parasympathetic responses could explain the finding of potential nucleus tractus solitari activation previously reported in three women with $\mathrm{SCls}$ during orgasm. ${ }^{19}$

There are many limitations to these data and those of others with regard to neural imaging of $\mathrm{SCl}$ sexual responses. First, the methods of fMRI analysis are constantly evolving; therefore, this study bears replication with new imaging techniques. Second, these data are limited regarding actual level of arousal of subjects and concomitant peripheral autonomic responses. Moreover, all of our subjects were several years after injury, and one of our subjects was on baclofen and ditropan, which could have altered her responses. Perhaps most importantly, the number of subjects in this report and that of other researchers ${ }^{18}$ is limited and thus results cannot be generalized. Owing to the multiple issues involved with imaging of sexual techniques, guidelines from the Third International Consultation on Sexual Medicine recommend that 10-20 subjects be assessed for sexual imaging studies in women. ${ }^{19}$ Finally, to comprehensively assess the neurophysiology of sexual responses in women with $\mathrm{SCls}$, it would be ideal to have imaging data for larger groups of subjects who have undergone both brain and spinal cord imaging.

Despite these limitations, our data provide baseline information to document the use of fMRI to study spinal sexual responses in women with $\mathrm{SCls}$. On the basis of this information, we propose repetition of this protocol in conjunction with observation of the brain responses during sexual stimulation in women with complete injuries above the level of T6. These women would presumably have an inability to transmit psychogenic neurological input to their thoracolumbar cord. Moreover, we suggest similar studies of those with complete cauda equina injuries with no preservation of lumbar or sacral reflex function. Detailed assessment comparing the remaining neurological and autonomic functions of women with incomplete SCls with functional MRI will help elucidate the neurological activity occurring in these women. Finally, use of spinal fMRI presuccessful and postsuccessful therapeutic intervention to treat sexual dysfunction would assist in determining the neurological changes associated with the results of intervention.

\section{ACKNOWLEDGEMENTS}

This study was supported by a grant from the Craig Nielsen Foundation Grant no. 2786.

\section{COMPETING INTERESTS}

The authors declare no conflict of interest.

\section{REFERENCES}

1 Anderson K. Targeting recovery: priorities of the spinal cord injured population. J Neurotrauma 2004; 21: 1371-1383.

2 Sipski ML, Alexander CJ. Sexual activities, response and satisfaction in women pre- and post-spinal accord injury. Arch Phys Med Rehabil 1993; 74: 1025-1029.

3 Sipski ML, Alexander CJ, Rosen RC. Physiologic parameters associated with psychogenic sexual arousal in women with complete spinal cord injuries. Arch Phys Med Rehabil 1995; 76: 811-818.

4 Sipski ML, Alexander CJ, Rosen RC. Physiologic parameters associated with sexual arousal in women with incomplete spinal cord injuries. Arch Phys Med Rehabil 1997; 78: 305-313.

5 Sipski ML, Alexander CJ, Rosen RC. The neurologic basis of sexual arousal and orgasm in women: effects of spinal cord injury. Ann Neurol 2001; 49: 35-44.

6 Alexander MS, Biering-Sorensen F, Elliott S, Kreuter M, Sonksen J. International spinal cord injury female sexual and reproductive function basic data set. Spinal Cord 2011; 49: 787-790.

7 Alexander MS, Biering-Sorensen F, Bodner D, Brackett NL, Cardenas D, Charlifue S et al. International standards to document remaining autonomic function after spinal cord injury. Spinal Cord 2009; 47: 36-43.

8 Wiedey J, Sipski Alexander M, Marson L. Spinal neurons activated in response to pudendal or pelvic nerve stimulation in female rats. Brain Res 2008; 1197: 106-114.

9 Cai RS, Sipski Alexander M, Marson L. Activation of somatosensory afferents elicit changes in vaginal blood flow and the urethrogenital reflex via autonomic efferents. J Urol 2008; 180: 1167-1172.

10 Kozyrev N, Figley CR, Alexander M, Richards JS, Bosma RL, Stroman PW. Neural correlates of sexual arousal in the spinal cords of able-bodied men: a spinal fMRI investigation. J Sex Mar Ther 2012; 38: 418-435.

11 Alexander MS, Kozyrev N, Bosma R, Figley CR, Richards S, Stroman P. FMRI localization of spinal cord processing underlying female sexual arousal. J Sex Mar Ther 2016; 42: 36-47.

12 Kozyrev N, Leham MN, Coolen LM. Activation of gastrin-releasing peptide receptors in the lumbosacral spinal cord is required for ejaculation in male rats. J Sex Med 2012; 9: 1303-1318.

13 Kreuter M, Taft C, Siosteen A, Biering-Sorensen F. Women's sexual functioning and sex life after spinal cord injury. Spinal Cord 2011; 49: 154-160.

14 Sipski M, Alexander CJ, Gomez-Marin O. Effects of level and degree of spinal cord injury on male orgasm. Spinal Cord 2006; 44: 798-804.

15 Sipski M, Alexander C, Gomez-Marin O, Spalding J. The effects of spinal cord injury on psychogenic sexual arousal in males. J Urol 2007; 177: 247-251.

16 Chehensse C, Bahrami S, Denys P, Clement P, Bernabe J, Giuliano F. The spinal control of ejaculation revisited; a systematic review and meta-analysis of anejaculation in spinal cord injured patients. Hum Reprod Update 2013; 19: 507-526.

17 Georgiadis JR, Simone Reinder AAT, Paans AMJ, Renken R, Kortekaas R. Men versus women on sexual brain function: prominent differences during tactile genital stimulation, but not during orgasm. Hum Brain Mapp 2009; 30: 3089-3101.

18 McLachlan EM. Diversity of sympathetic vasoconstrictor pathways and their plasticity after spinal cord injury. Clin Auton Res 2007; 17: 6-12.

19 Salonia A, Giraldi A, Chivers ML, Georgiadis JR, LevinR, Maravilla KR et al. Physiology of women's sexual function: basic knowledge and new findings. J Sex Med 2010; 7: 2637-2660. 